tion, ${ }^{4}$ and with a similar "intellectual content," were not preferred by the respondents, largely for aesthetic reasons. The greater convenience .nd economy of reprints cited by several of those who returned the questionnaire no doubt reflected a personal outlook not shared, I imagine, by most reprint providers-although I hope that most would support the solitary philatelist.

If the number of reprint requests reflects the interest generated, it seems an author ought to receive over 110 requests for a paper of above average interest. Most authors accept a moral responsibility to provide reprints and this must be balanced against the costs. Often these are wholly or partially derived from research funds, official sources, or the NHS. In any case, money has to be spent and if borne directly by the individual concerned, as in my case, almost certainly would be better spent on more worthwhile projects or persons. ${ }^{5}$

${ }^{1}$ Hamilton-Miller, J M T, British Medical fournal, 1977, 1, 509.

2 Pickles, V R, British Medical fournal, 1977, 1, 510.

3 Welsby, P D, Practitioner, 1977, 218, 382.

4 Welsby, P D, British fournal of Occupational Therapy, 1976, 39, 198.

${ }^{5}$ Welsby, J B, personal communication, 1977.

(Accepted 26 fanuary 1978)

Department of Infectious Diseases, Royal Free Hospital, Coppetts Wood Hospital, London N10 1JN

PHILIP D WELSBY, MRCP, senior registrar

\section{Trial of aspirin during weaning patients with transient ischaemic attacks from anticoagulants}

Treatment with anticoagulants reduces both the frequency of transient ischaemic attacks (TIAs) and the risk of cerebral infarction. ${ }^{2}$ One of its problems is recurrence of TIAs and cerebrovascular accidents during the three months after treatment is stopped. ${ }^{3+}$ Recent work has suggested that, because of its effect on platelets, aspirin may reduce the frequency of strokes and TIAs. ${ }^{5}$ The present study explores the role of aspirin during withdrawal of anticoagulant treatment.

\section{Methods and results}

Eighteen patients who had been taking warfarin for at least a year because of TIAs were included. Twelve had had attacks in the carotid (10 hemisphere, two retinal) and six in the vertebrobasilar territory. Thirteen had undergone angiography showing appropriate vascular lesions. After explanation the patients were divided into two groups. Six in each group had had carotid and three vertebrobasilar TIAs. Warfarin was withdrawn from both groups but one group received aspirin, $300 \mathrm{mg}$ twice daily, while the other received inert tablets of identical appearance. The patients were seen every two weeks for three months by a physician who was unaware to which group they belonged.

The attack rate while taking warfarin was 0.02 attacks per patient months (24 attacks in 981 patient months). Three patients had experienced attacks at a time when warfarin had been temporarily reduced. Only one patient had had an attack in the three months before the start of the study. In the three months after warfarin was stopped six patients experienced a total of eight TIAs, the TIA occurring in the same territory as the initial episode. If the three months before and after stopping warfarin are compared, there is a significant increase in the proportion of patients experiencing TIAs $\left(\chi^{2}-4.01 \mathrm{P}<0.05\right)$. Warfarin was restarted within the study period in two of the patients who experienced a recurrence (one at four and the other at seven weeks). The attack rate calculated for the period off warfarin was 0.16 attacks per patient month (eight TIAs in 50.75 patient months). This represents an eightfold increase over the preceding three months on warfarin $(0.02$ attacks per patient month). There was no difference between the group receiving aspirin and that receiving placebo, three patients in each group having further episodes.

\section{Discussion}

The importance of TIAs lies in the increased risk of a stroke, which over five years is roughly one in three. Most TIAs are believed to be due to thromboembolism from carotid lesions. Endarterectomy and anticoagulant treatment are effective in reducing the frequency of
TIAs and the risk of strokes. Although anticoagulants are effective, there are difficulties in their use. Marshall and Reynolds ${ }^{3}$ showed that there was an increase in ischaemic events when anticoagulants were withdrawn. We confirmed this in the present study, the attack rate showing an eightfold increase.

There is currently considerable interest in the use of aspirin for treating TIAs through its inhibitory effect on platelet aggregation. ${ }^{5}$ On the basis of present information it was appropriate to see whether aspirin would protect patients against the increased risk of thromboembolic incidents during the period immediately after stopping warfarin treatment. The results showed no protective value from aspirin, $300 \mathrm{mg}$ twice daily. This may not be entirely relevant to the general use of aspirin in patients with TIAs, since there may be a unique hypercoagulable state during withdrawal of anticoagulation. Nevertheless, we noted that in each case the TIAs that occurred on withdrawal were in the same territory as the patients' spontaneous episodes, which suggests that the pathophysiological mechanism was still the original one.

Thus, while the roles of surgery and anticoagulants are generally defined, that of aspirin is only now emerging. A more rational choice of treatment for TIAs may be possible when we can detect the presence, source, and nature of embolic material in individual patients better than at present.

We thank Dr R O McKeran for his help in following some of the patients during this study, Seaford Laboratories for a supply of placebo tablets, and Miss Beryl Laatz for maintaining the records of the trial.

${ }^{1}$ Millikan, C H, Stroke, 1971, 2, 201.

2 Whisnant, J P, Matsumoto, N, and Elveback, L. R, Mayo Clinic Proceedings, 1973, 48, 894 .

${ }^{3}$ Marshall, J, and Reynolds, E H, Lancet, 1965, 1, 5

${ }^{4}$ Marshall, J, Circulation, 1963, 28, 329.

5 Fields, W S, Lemak, N A, Frankowski, R F, and Hardy, R J, Stroke, 1977, $\mathbf{8}, 301$.

(Accepted 26 fanuary 1978)

Institute of Neurology, National Hospital for Nervous Diseases, London WC1N 3BG

J JESTICO, MRCP, registrar

M J G HARRISON, DM, FRCP, consultant physician

JOHN MARSHALL, MD, FRCP, professor of neurology

\section{Unusual case of skin discoloration}

Cyanosis is recognised as a deep blue discoloration of the skin due to increased amounts of reduced haemoglobin in the blood stream. This is particularly evident in the exposed parts of the body such as the face and the fingers and toes. Sulphaemoglobinaemia and methaemoglobinaemia simulate this condition and are usually the effect of drug interference with the haemoglobin molecule. A metallic blue colour is a feature of the absorption of silver, which is particularly striking in its response to ultraviolet light. A somewhat similar colour is seen in cases of haemochromatosis.

We describe here a patient, dubbed "the blue lady", whose remarkable appearance remained a puzzle for many months.

\section{Case report}

A 51-year-old nurse had noticed that her lips, face, and trunk had developed a greyish-blue colour which seemed less definite on her fingers and toes. She was a garrulous woman with a long history of psychiatric ailments which had apparently followed poliomyelitis when young, since this had left her severely disabled in spite of numerous operations to correct the deformity of her right leg. The results of all routine tests of the cardiovascular, respiratory, and haematological systems were normal including the arterial blood gases. Spectrographic tests for methaemoglobinaemia and sulphaemoglobinaemia gave negative results. It was noted, however, that the blood had a brown tinge, whereas the plasma was pink. On precipitation of the protein, the supernatant fluid became clear. Scrutiny of many urine specimens showed that it was often dark yellow, though not concentrated. The $\mathrm{pH}$ was acid, but when made alkaline the urine changed to an intense purple-blue. Specific inquiries regarding drugs which might contain a dye showed that she had been taking imipramine hydrochloride and diazepam for six years, but denied completely using gentian violet or methyl violet. Subsequently 